\title{
Mesures électrochimiques appliquées à l'étude de la protection cathodique des ouvrages de la Rance
}

\author{
The application of electro-chemical \\ measurements to a study of cathodic protection \\ for the various works in the Rance project
}

\section{R. LEGRAND}

CHEF DE LA DIVISION ESSAIS ET PROTEGTION DES OUYRAGES

\section{PAR}

ET

DE GAz DE FRANCJ:
Rappel des principes théoriques de la protection cathodique.

Résultats des essais entrepris pour déterminer les caractéristiques des alliages inoxydables placés dans des conditions électrochimiques voisines de celles qui leur seront imposées in situ, soit à cause des impératifs de construction, soit sous l'influence de la protection cathodique. Comparaison avec les mesures effectuées sur le groupe de Saint-Malo.

Conclusions pratiques.

\begin{abstract}
A review of the theoretical principles of cathodic protection.

Results of tests carried out to determine the characteristics of stainless alloys under similar electro-chemical conditions to those likely to affect them on the site, either due to constructional requirements or to calhodic protection. Comparison of these results with measured data from the Saint-Malo nnit.

practical conclusions.
\end{abstract}

Le service Essais et Protection des Ouvrages, de Gaz de France, assure depuis 1947 les études de protection cathodique appliquées aux installations d'Electricité et de Gaz de France.

A ce titre, lors de la constitution du Comité Corrosion du barrage de la Rance, il a reçu pour mission d'étudier la protection cathodique en vue de son application aux ouvrages immergés.

Les travaux relatifs à l'expérimentation de la protection cathodique sur le groupe expérimental de Saint-Malo ont été effectués en collaboration avec la Région d'Equipement Marémotrice.

L'expérimentation sur éprouvettes en eau de mer «vivante» tut poursulve avec le concours du Service d'Etudes pour l'Utilisation des Marées.

Rappelons, avant d'aborder le problème particulier des ouvrages de la Rance, les bases fondamentales de la protection cathodique. Cellesci reposent, pour une bonne part, sur les travaux du professeur Pourbaix.

A partir de la théorie de la pression de dissoIution de Nernst et des lois d'équilibre physicochimique, Pourbaix a établi l'équivalence entre 
le travail chimique et le travail électrique correspondant selon l'équation :

$$
\begin{aligned}
\mathrm{E} & =\mathrm{E}_{0}+\frac{0,059}{n} \times \log (\mathrm{M}) ; \\
\mathrm{E}^{0} & =\frac{\Sigma \nu \mu^{0}}{23060 n} ;
\end{aligned}
$$

$E$ est le potentiel d'équilibre de la réaction;

$\mathrm{E}^{0}$ est la tension d'équilibre normale, cas particulier où tous les corps sont à l'état standard;

$\mu^{0}$ est l'enthalpie de formation normale;

$\checkmark$ le coefficient stoechiométrique;

$n$ le nombre d'électrons entrant en jeu dans la réaction;

M l'activité ou la fugacité des corps réactionnels.

Les développements des données que nous venons de résumer brièvement ont conduit à établir, pour chacun des corps simples, les domaines de stabilité de ces corps et de chacun des composants qu'ils peuvent former avec l'eau, en fonction du potentiel et $\mathrm{du} \mathrm{pH}$.

La figure 1 représente le diagramme thermodynamique du fer. Les lignes de séparation entre le fer et ses produits de corrosion correspondent à une concentration de $10^{--6}$ atome gramme de fer par litre; on voit que, lorsque le fer se trouve dans des circonstances de potentiel et de $\mathrm{pH}$ le plaçant dans le domaine électronégatif,

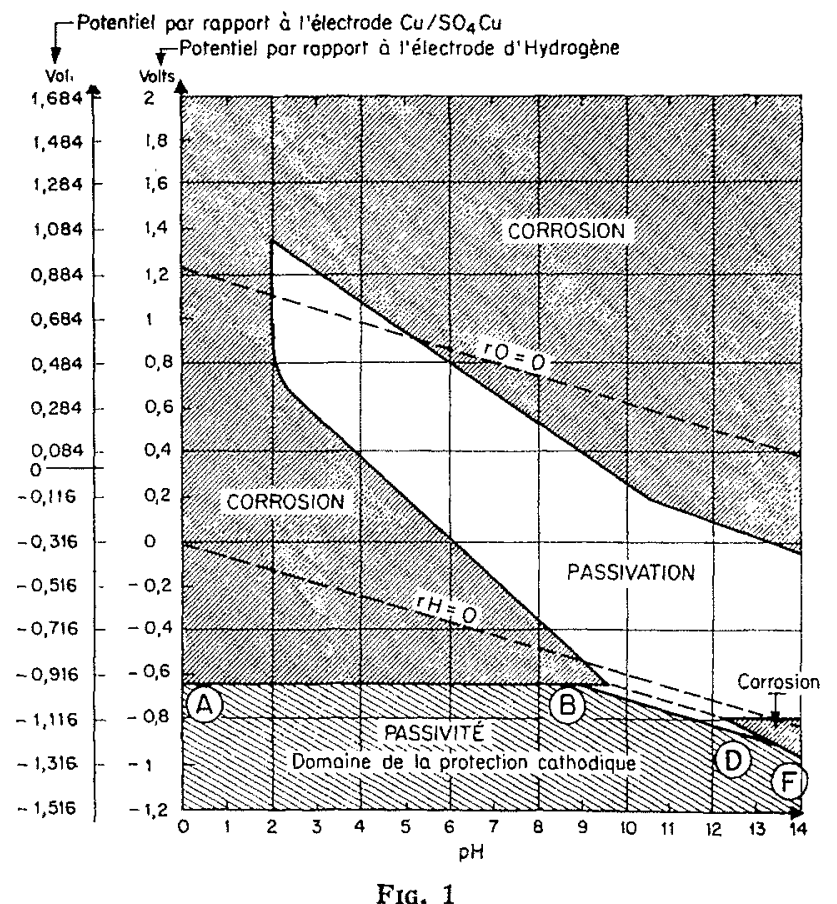

Diagramme de corrosion.

Système fer-cau (d'après le professeur Pourbaix). dit domaine d'immunité, limité par la ligne A.B.D.F., toute réaction entre le fer et le milieu aqueux est thermodynamiquement impossible.

Le but de la protection cathodique consiste donc à placer, par une polarisation imposée, le métal à une valeur de potentiel telle qu'il se trouve dans le domaine d'immunité.

Cette polarisation est obtenue en faisant débiter une source de courant continu entre la structure à protéger et des anodes isolées, placées dans le milieu agressif considéré.

Le problème de la protection cathodique de l'acier ordinaire dans l'eau de mer ne présente aucune difficulté théorique particulière.

Il est seulement nécessaire d'assurer une bonne répartition des anodes afin d'obtenir, sur la surface à protéger, une distribution homogène de la densité de courant.

Cette dernière doit être compatible avec une bonne tenue de la peinture. On sait, en effet, que l'application d'une polarisation électronégative peut conduire, à partir d'un certain seuil de tension, à une accélération du phénomène de cloquage se produisant sur les films de peinture.

Les questions particulières à résoudre pour trouver une solution complète d'anticorrosion des ouvrages de la Rance seront exposées ciaprès.

Elles sont principalement dues à l'association des matériaux de diverses natures en contact avec l'eau de mer.

Les ouvrages immergés comprennent, en effet, successivement :

- la conduite amont en béton armé,

- le corps du groupe en acier ordinaire,

- la roue, la ceinture et le distributeur en alliages inoxydables suivis du conduit aval en béton armé.

Comment envisager la protection de cet ensemble?

Un traitement orthodoxe du problème nécessiterait l'isolement électrique des différents matériaux entre eux; il serait alors possible de les protéger séparément en fonction de leurs caractères propres.

Techniquement, cette méthode semble peu réalisable; en effet, l'expérience a montré qu'il était difficile d'établir et de maintenir un bon isolement électrique entre des organes simples enterrés. A fortiori, il paraît problématique d'obtenir un tel isolement sur un ouvrage de la taille et de la complexité d'un groupe marémoteur.

Nous devons donc considérer que les différentes parties de l'ouvrage formeront un ensemble électriquement continu. 
Cet ensemble peut se présenter électrochimiquement sous deux aspects :

$1^{\circ}$ La protection contre l'agressivité de l'eau de mer est assurée par l'application de peintures et l'utilisation d'alliages inoxydables, en l'absence de toute polarisation extérieure;

$2^{\circ}$ Les systèmes précédents sont complétés par une polarisation cathodique.

- Dans le premier cas, comme l'ont montré les travaux antérieurs (1) l'association, en présence d'eau de mer, de l'acier au carbone avec un bronze ou un acier inoxydable donne naissance à un couple de corrosion notable. Le couple de corrosion ainsi formé provoque une polarisation cathodique de l'alliage inoxydable, alors que l'acier ordinaire anodique se dissout suivant les lois de Faraday.

- Dans le second cas, une circulation de courant entre les anodes isolées du groupe et ce dernier assure la protection cathodique de l'acier ordinaire en le portant à un potentiel inférieur à $-850 \mathrm{mV}$. Les valeurs de potentiel indiquées dans cet exposé sont mesurées à l'aide d'une électrode au calomel — CIK saturé.

En aucune circonstance, l'alliage inoxydable n'est dans les conditions de potentiel qu'il occupe seul spontanément.

Il est donc nécessaire de connaitre le comportement, vis-àvis de l'eau de mer, des métaux inoxydables polarisés cathodiquement.

Généralement, les travaux effectués sur ces alliages et, en particulier sur les aciers inoxydables, ont été conduits dans le domaine anodique où apparaissent les propriétés protectrices des couches passivées de ces métaux qui sont à la base de leur utilisation habituelle.

Devant le peu d'informations recueillies dans la bibliographie concernant les études dans le domaine cathodique, nous avons été amenés à entreprendre des essais de laboratoire à caractères généraux sur les réactions des alliages dans ce domaine.

Nous avons, d'autre part, à partir de novembre 1960, appliqué la protection cathodique sur le groupe expérimental de Saint-Malo.

\section{ESSAIS DE LABORATOIRE}

Deux types d'essais ont été réalisés :

-- D'une part, une expérimentation de longue durée sur des éprouvettes soumises à des densités de courant constantes en fonction du temps;

- D'autre part, nous avons tracé les courbes de polarisation, en commandant le potentiel des matériaux étudiés à partir des alliages inoxydables envisagés pour la Rance, d'un régulateur électronique dont on faisait varier la tension pilote.

\section{Essais de longue durée}

Des éprouvettes, immergées durant 125 jours dans de l'eau de mer «vivante», ont été polarisées à courant constant. Les conditions mêmes de l'expérience imposaient l'implantation d'essais en bordure de mer.

(1) J. Changarnier et R. Legrand: Utilisation des courbes de polarisation pour étudier la tenue en eau de mer des aciers inoxydables et des bronzes. Rapport $\mathrm{n}^{\circ} 9$ bis, Ves Journées de l'Hydraulique, S.H.F., Aix-enProvence, 1958.
Les figures 2 et 3 représentent l'installation réalisée à Saint-Servan dans les locaux du S.E.U.M.

Les éprouvetles ont été usinées sous forme d'haltères plates de profil homothétique au profil normalisé. Cette disposition nous a permis, en un seul essai, de mesurer :

la perte de poids, la résistance à la traction,

la striction des matériaux étudiés.

Les densités de courant retenues pour l'expérimentation furent de $0,40,80,150,220,360$, $900,5000,20000$ et $90000 \mathrm{~mA} / \mathrm{m}^{2}$.

Le bac à niveau constant dans lequel ces éprouvettes étaient disposées recevait un apport permanent d'eau de mer puisće dans le sas de l'écluse de Saint-Malo.

Après 125 jours, les échantillons ont été pesés.

Les pertes de poids représentées sur la figure 4.

Dans la partie gauche des courbes, les résultats ont été faussés par une corrosion due à l'aération différentielle produite par le vernis destiné à isoler les têtes d'éprouvettes.

Nous avons repris ces essais sous faible densité (fig. 5). 


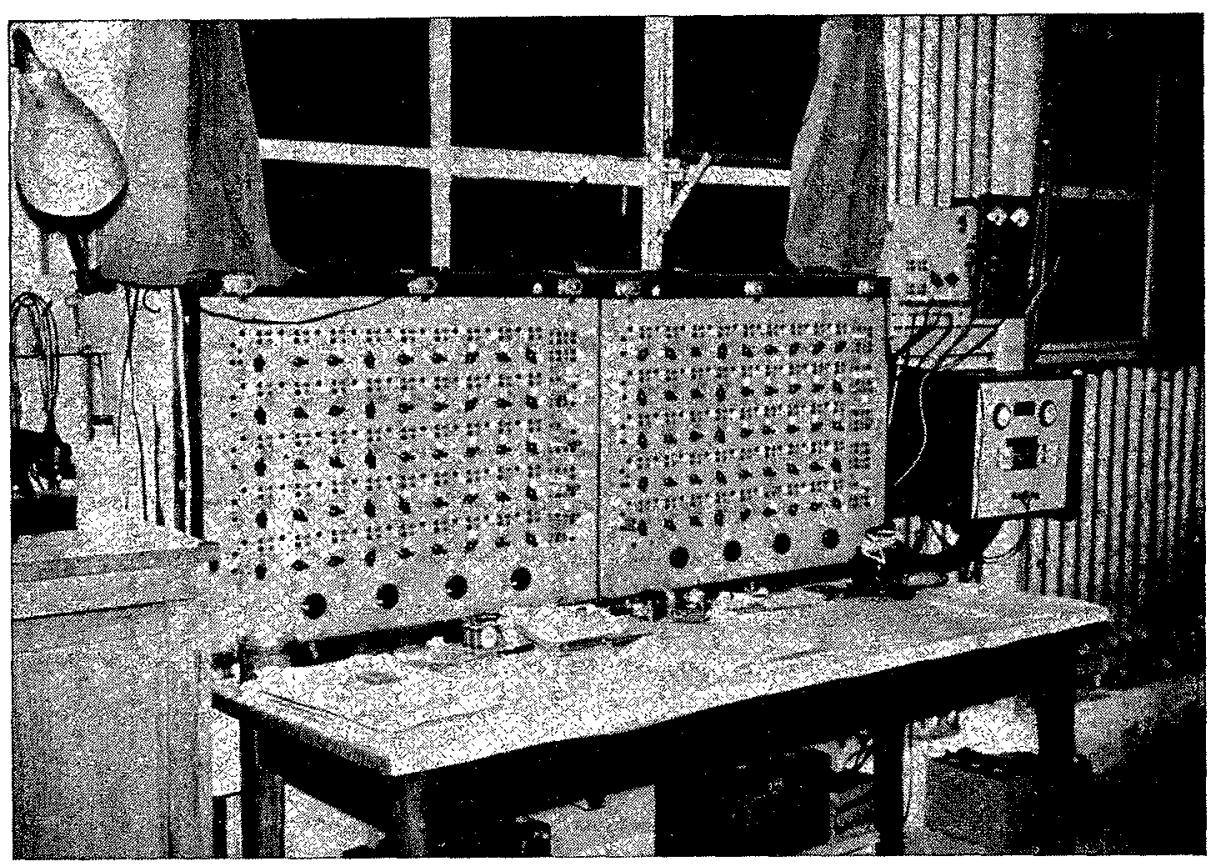

Fig. 2

Tableau de réglage des intensités.

L'examen des résultats montre que seuls ceux des aciers dont la teneur est supérieure à $15 \%$ de chrome et $4 \%$ de nickel restent inattaqués en eau de mer tant à l'état de témoin que soumis à une polarisation cathodique.
Les cupro-aluminiums résistent très bier jusqu'à $5000 \mathrm{~mA} / \mathrm{m}^{2}$; au-delà de cette valeur ils subissent une désaluminisation provoquér par l'élévation du $\mathrm{pH}$ de l'électrolyte au contac du métal. Ce phẻnomène n'intervient pas lors d

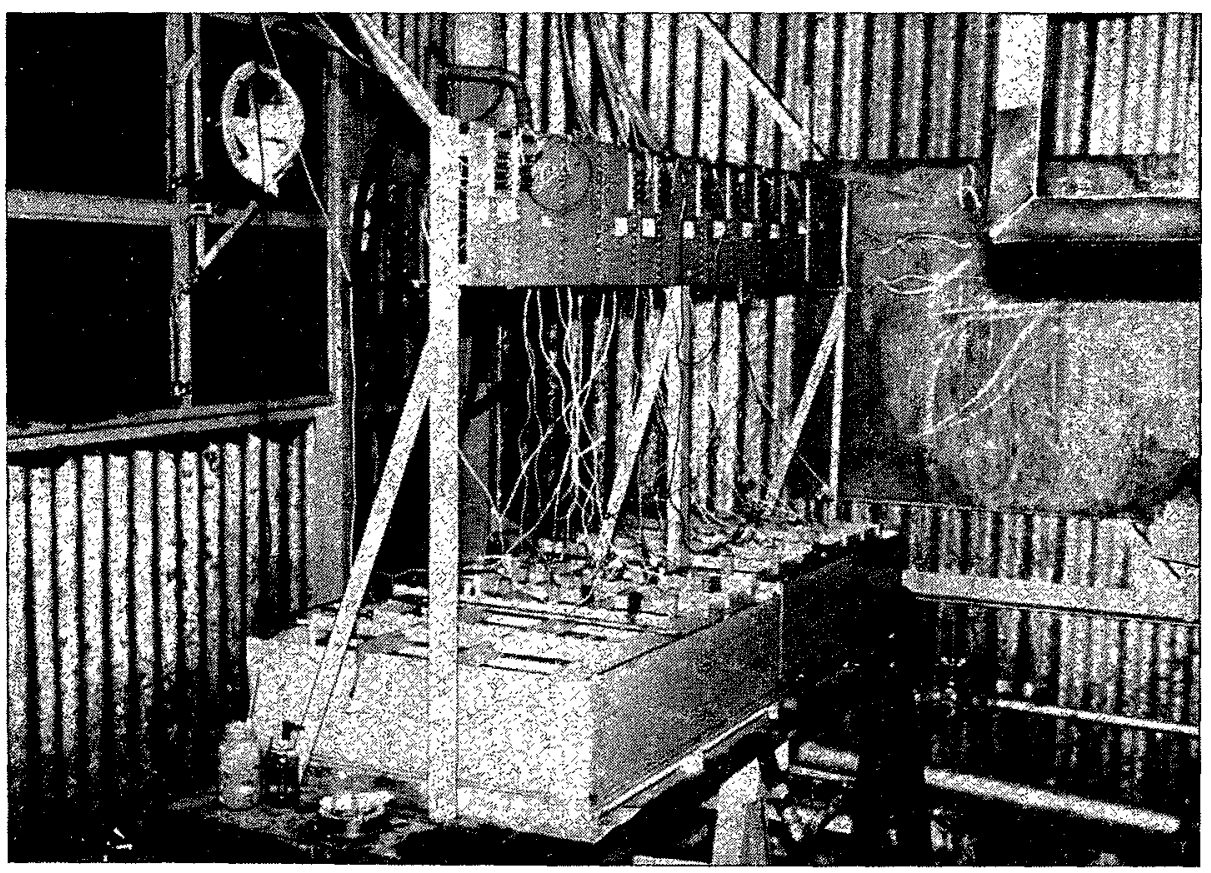

FIG. 3

Bac d'essai à niveau constant 


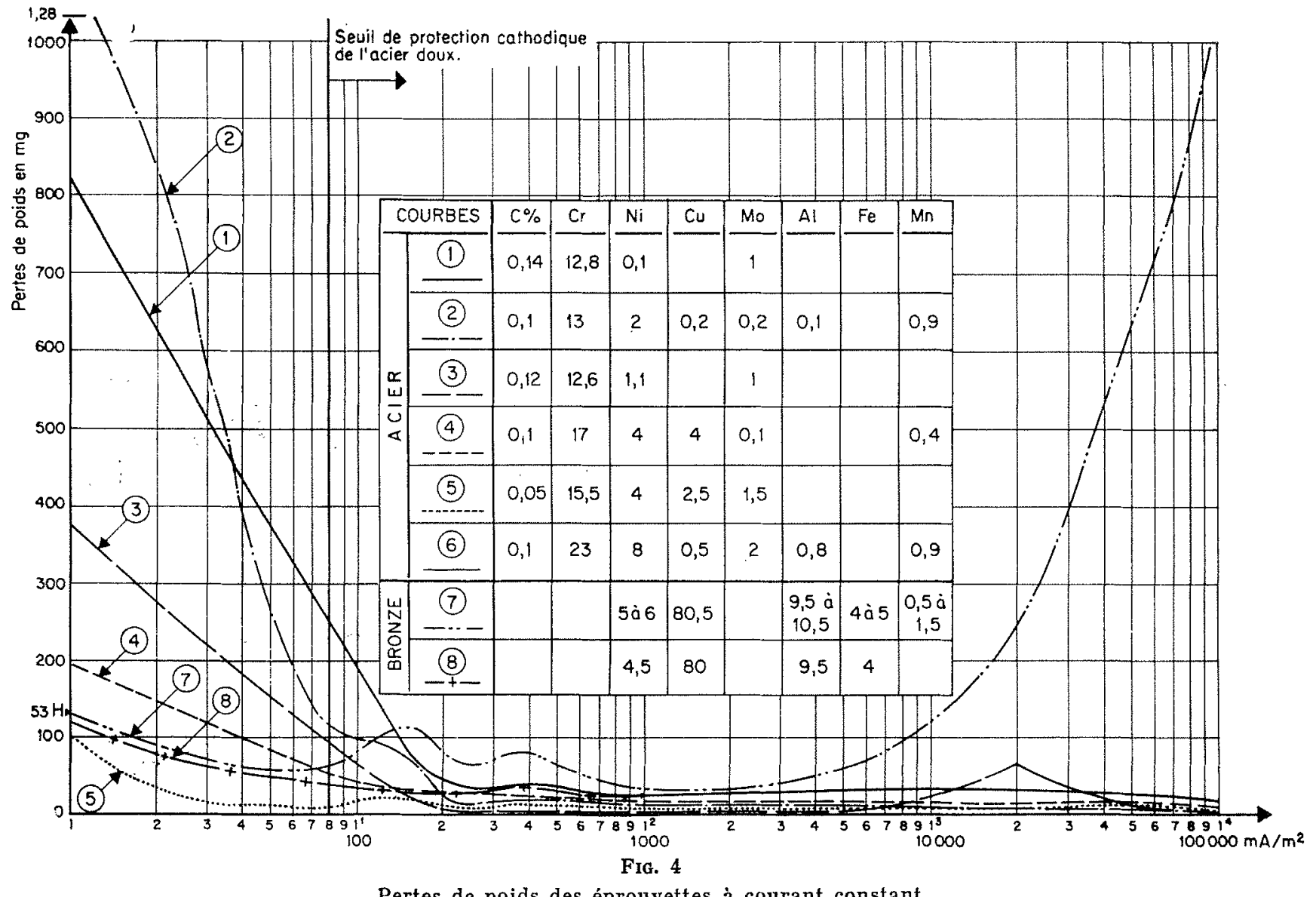

Pertes de poids des éprouvettes à courant constant.

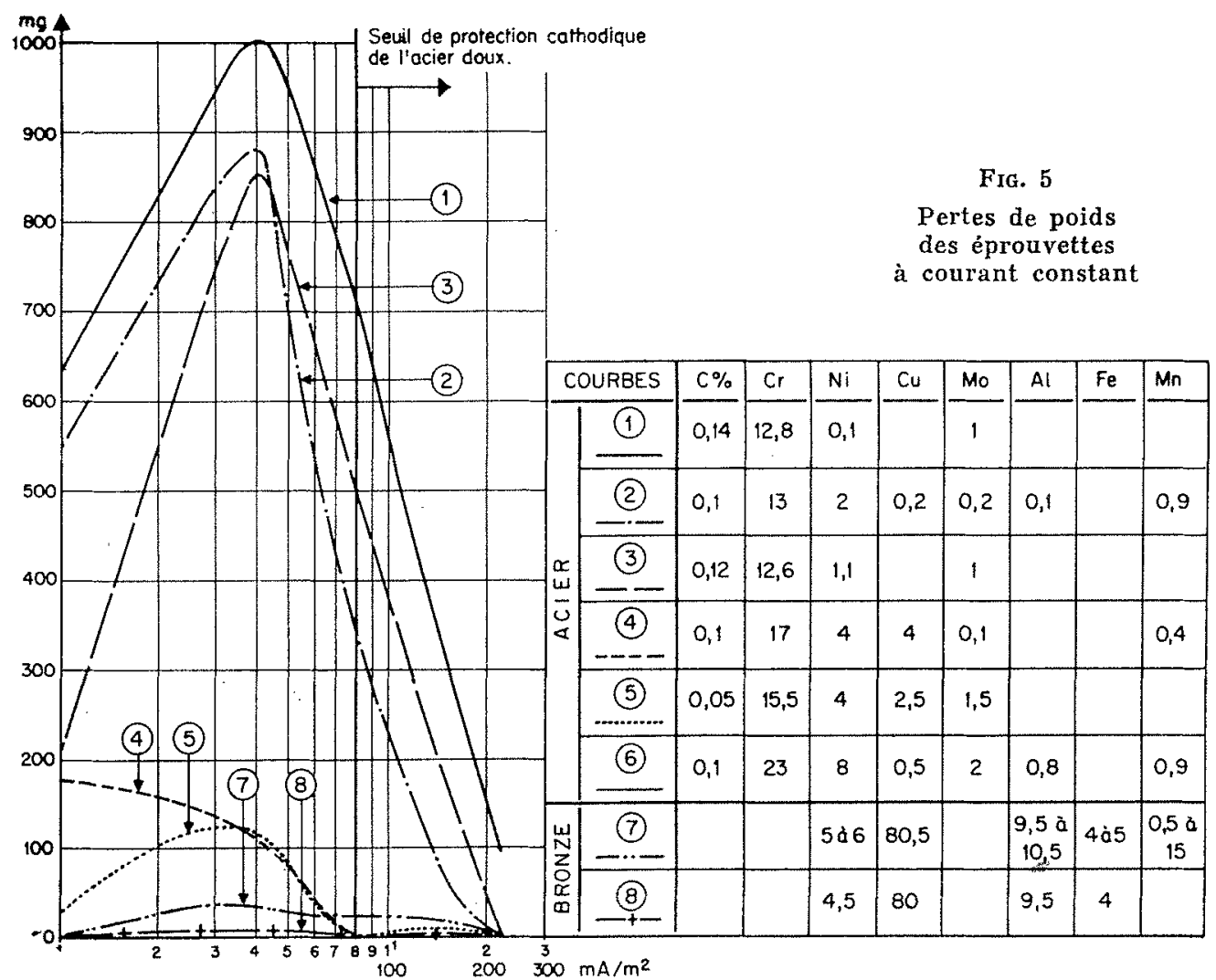


l'application de la protection cathodique, la densité de courant mise en œuvre étant toujours bien inférieure à $5000 \mathrm{~mA} / \mathrm{m}^{2}$.

Dans le domaine des faibles densités, les aciers dont la teneur en nickel et en chrome est respectivement inférieure ou égale à $15 \%$ et $4 \%$ présentent une attaque dont le maximum correspond à la densité de $40 \mathrm{~mA} / \mathrm{m}^{2}$.

Ceci peut s'expliquer si l'on considère la nature de l'inoxydabilité de ces alliages.

Celle-ci est due, non à la stabilité thermodynamique du matériau vis-à-vis de l'électrolyte, mais bien à une couche de passivité homogène, électriquement conductrice, qui inhibe les réactions d'oxydation.

La présence d'oxygène est nécessaire pour assurer la formation de cette couche. On conçoit donc que la polarisation cathodique agissant comme un réducteur diminue la stabilité de cette couche.

Si cette polarisation est insuffisante pour assurer la protection cathodique et si la concentration de l'alliage en constituants nobles est faible, une attaque se produira aux points où le métal est dépassivé.

Les variations de potentiel, en fonction $\mathrm{du}$ temps, d'un acier à $15 \% \mathrm{Cr}-4 \% \mathrm{Ni}-2,6 \% \mathrm{Cu}$ - 1,5\% Mo, sont représentées sur la figure 6 .

Le métal polarisé à courant constant se caractérise par deux états de stabilité :

- le premier dans un domaine de potentiel compris entre +150 et $-300 \mathrm{mV}$, correspondant à l'état passif.

- le second vers - $1100 \mathrm{mV}$, où le métal actif est protégé cathodiquement.

Les diagrammes potentiel - temps des autres alliages étudiés ont sensiblement la même allure.

Afin de déterminer l'incidence éventuelle de la polarisation cathodique sur les propriétés mécaniques des alliages, les échantillons on été conservés dans la neige carbonique, puis éprouvés à la traction sur machine Amsler après retour à la température ordinaire. Ces essais ont été effectués avec le concours du laboratoire de la Production thermique E.D.F.

Il résulte de ces expériences que la résistance à la traction rapportée à la section utile des éprouvettes n'est pas modifiée.

La détermination du coefficient de striction peut servir à évaluer l'influence fragilisante d'un chargement en hydrogène produit par électrolyse.

Le tableau I donne les valeurs du coefficient de striction en fonction de la nature des alliages et de la densité de courant. Les résultats obtenus sont assez dispersés, la mesure de la section, au droit de la rupture, sur des éprour
TABLEAU I

Coefficient de striction (\%) en fonction de la densité de courant

\begin{tabular}{|c|r|r|r|r|r|}
\hline AcIERs & 0 & $40 \mathrm{~mA}$ & $80 \mathrm{~mA}$ & $150 \mathrm{~mA}$ & $\begin{array}{r}220 \\
\mathrm{~mA} / \mathrm{m}^{2}\end{array}$ \\
\hline 1 & 29 & 28 & 40 & 29 & 2 \\
2 & 29 & - & 20 & 0 & 2 \\
3 & 33 & 40 & 30 & 27 & 3 \\
4 & 31 & 48 & 44 & 48 & 3 \\
5 & 46 & 39 & 42 & 2 & 3 \\
6 & 62 & 37 & 37 & 25 & 10 \\
\hline Bronzes & & & & & \\
\hline 7 & 8 & 12 & 12 & 16 & 16 \\
\hline 8 & 10 & 13 & 11 & 10 & 9 \\
\hline
\end{tabular}

vettes de section rectangulaire, étant assez imprécise.

On peut remarquer toutefois, que les aciers martensitiques et austénoferritiques présentent, à partir de 150 à $220 \mathrm{~mA} / \mathrm{m}^{2}$ une diminution importante de la striction. Les cupro-aluminiums et l'acier austénitique 18 - $11-3$ qui n'est pas porté ici, ne sont pas fragilisés sous les densités de courant que nous avons utilisées.

Il convient, d'ailleurs, de préciser l'influence de ce chargement en hydrogène. Comme l'ont montré P. Bastien et P. Azou (2), la fragilité due à l'hydrogène ne se manifeste pas lorsque l'acier est soumis à une vitesse de déformation supérieure à $8 \cdot 10^{-3} \mathrm{Nep} / \mathrm{s}$. La résistance au choc de l'acier n'est donc pas affectée par ce chargement. De même, les déformations élastiques ne sont pas modifiées, la fragilisation ne se produisant que dans Ie domaine plastique.

\section{Courbes de polarisation}

Les diagrammes de Pourbaix permettent, en fonction du potentiel métal-électrolyte et du $\mathrm{pH}$, de situer l'état thermodynamiquement stable du métal étudié. Mais, en dehors du domaine

(2) Influence de la vitesse et de l'amplitude des déformations plastiques sur le comportement de l'acier chargé en hydrogène. Journées de la Société francaise de Métallurgie, Paris, 25 octoble 1955. 


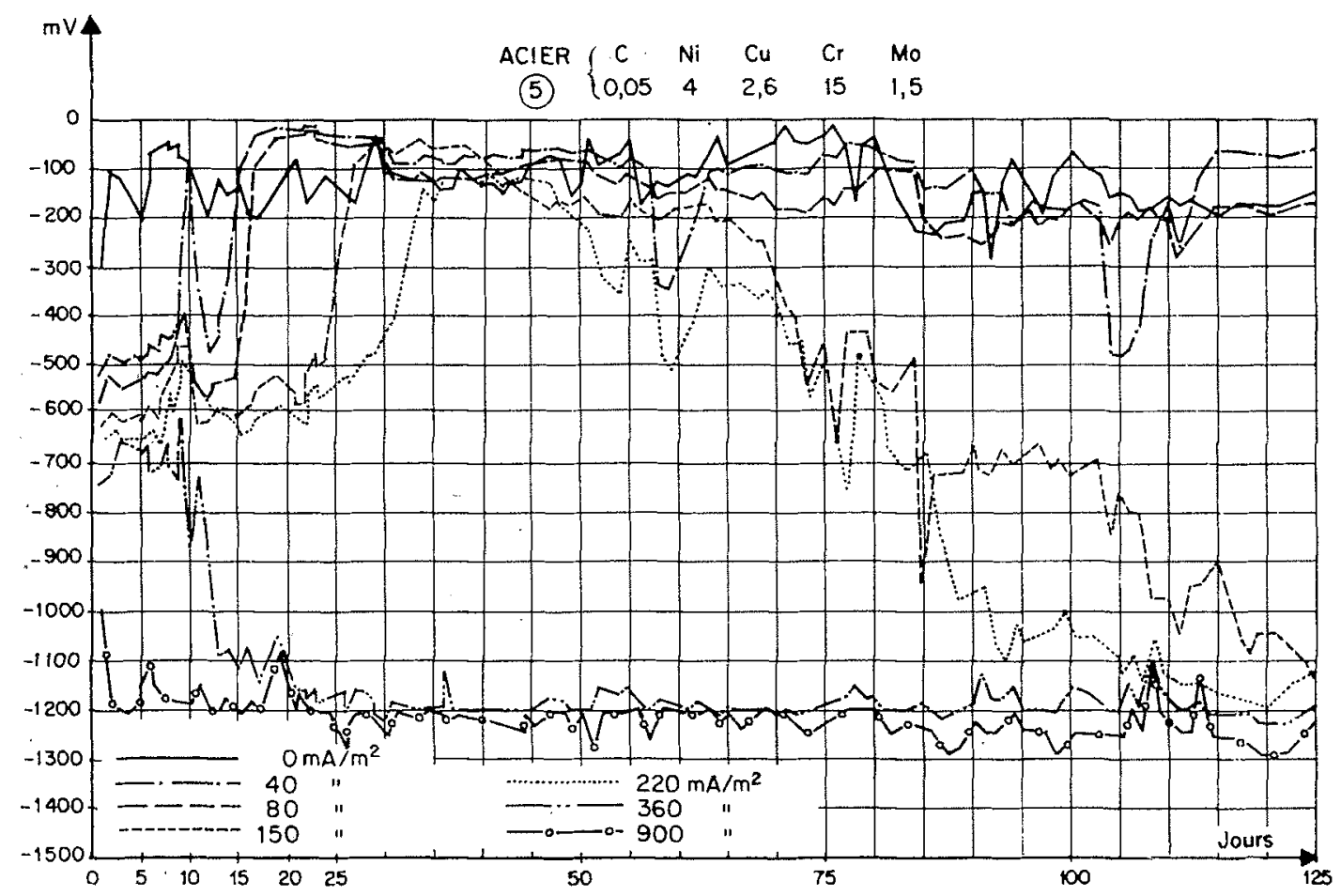

FIG. 6

Potentiel, en fonction du temps, d'éprouvettes soumises à intensité constante.

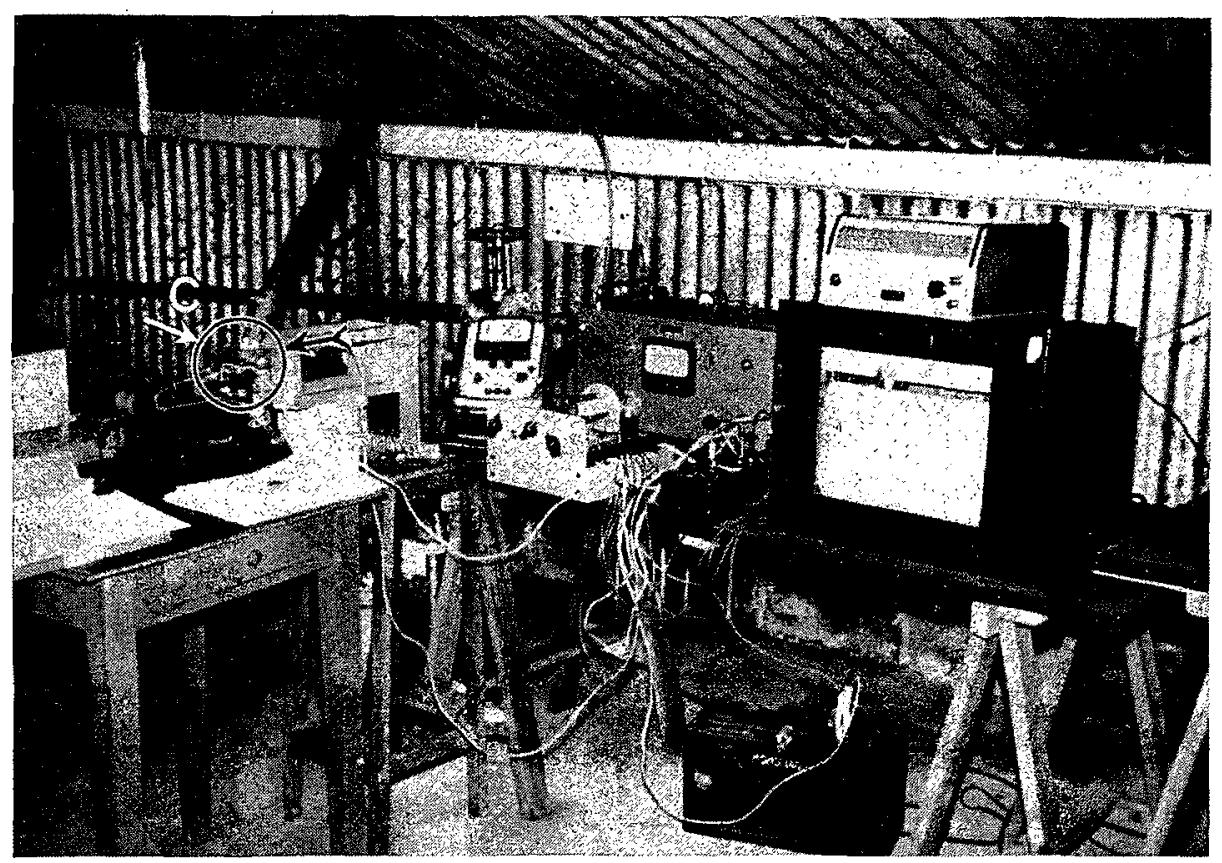

Fra. 7

Ensemble : cellule, enregistreur potentiostat avec commande motorisée pour tracé des courbes de polarisation. 


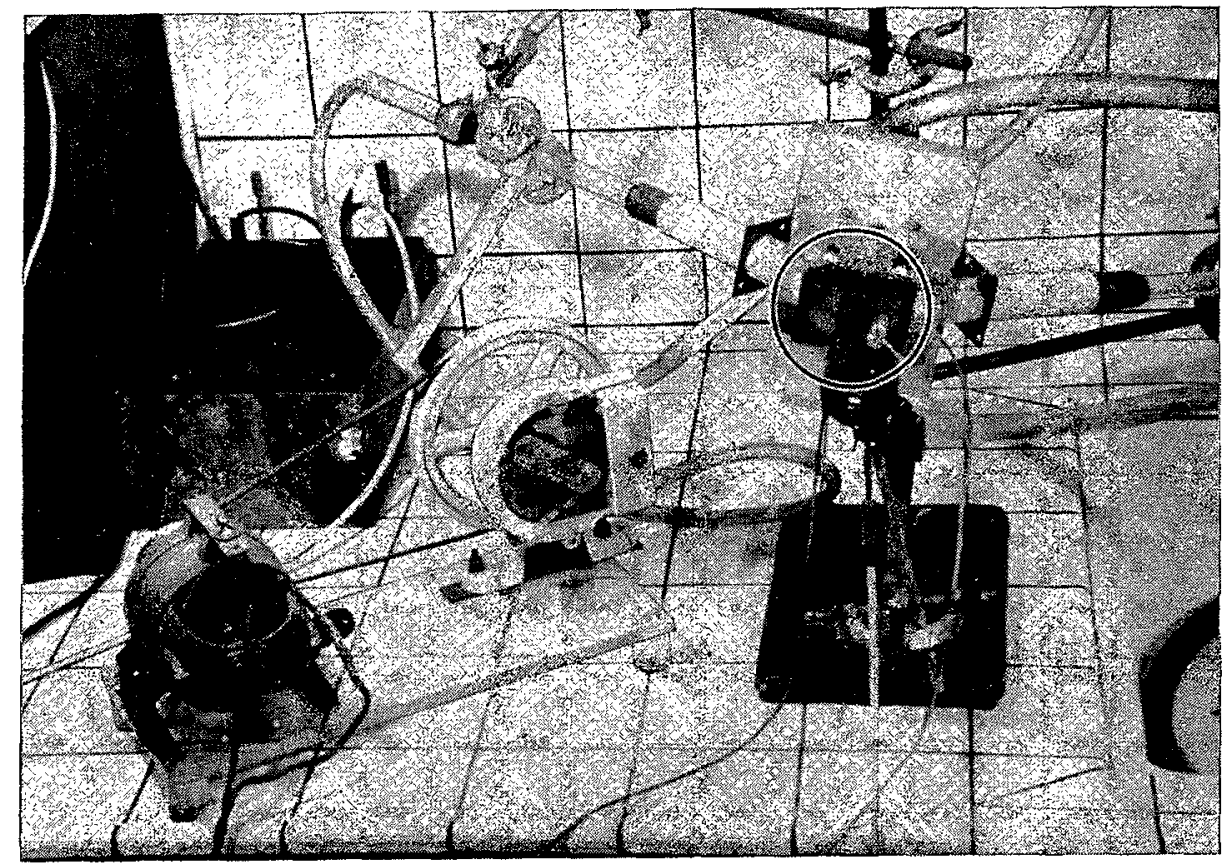

Fig. 8

Cellule d'essai et pompe de circulation.

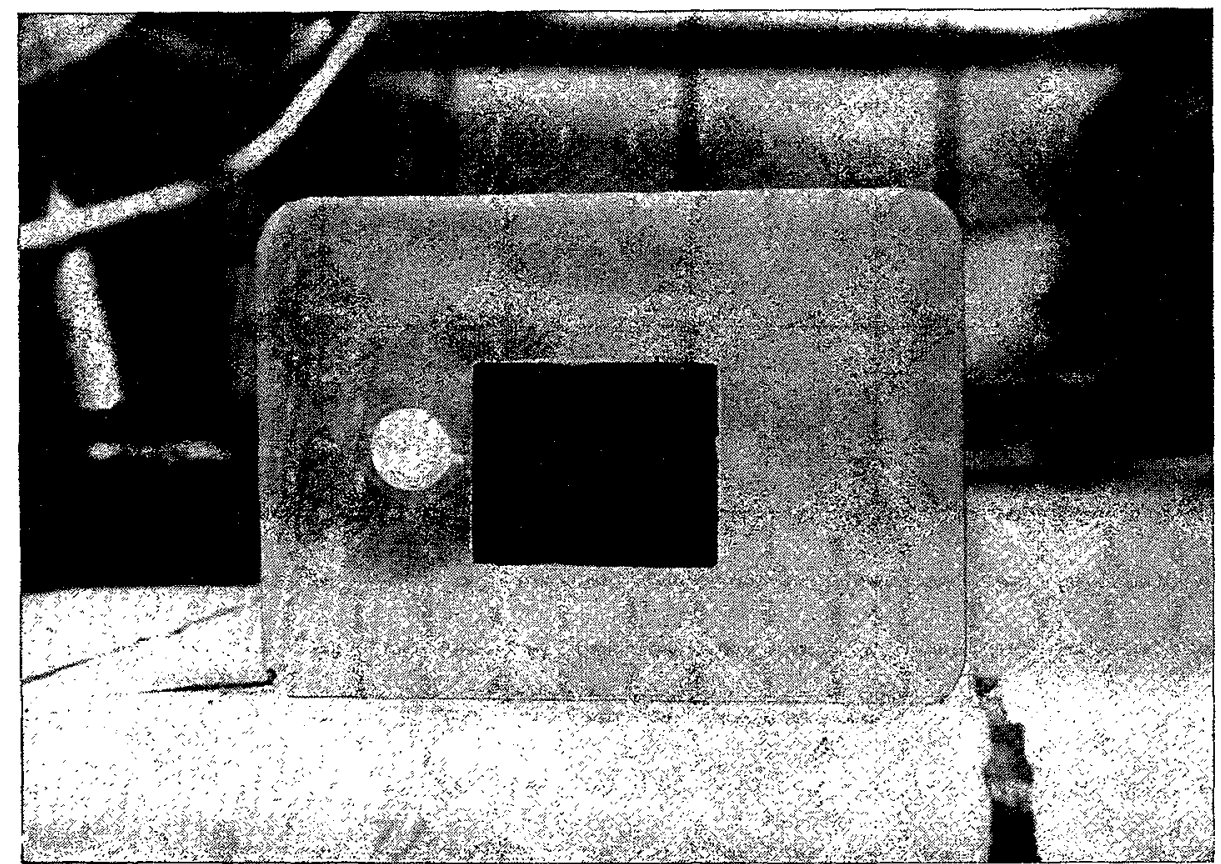

Frg. 9

Détail de l'éprouvette formant fermeture de la cellule d'essai. On distingue le trou de diamètre $0,5 \mathrm{~mm}$

servant à la communication avec l'électrode de référence. 


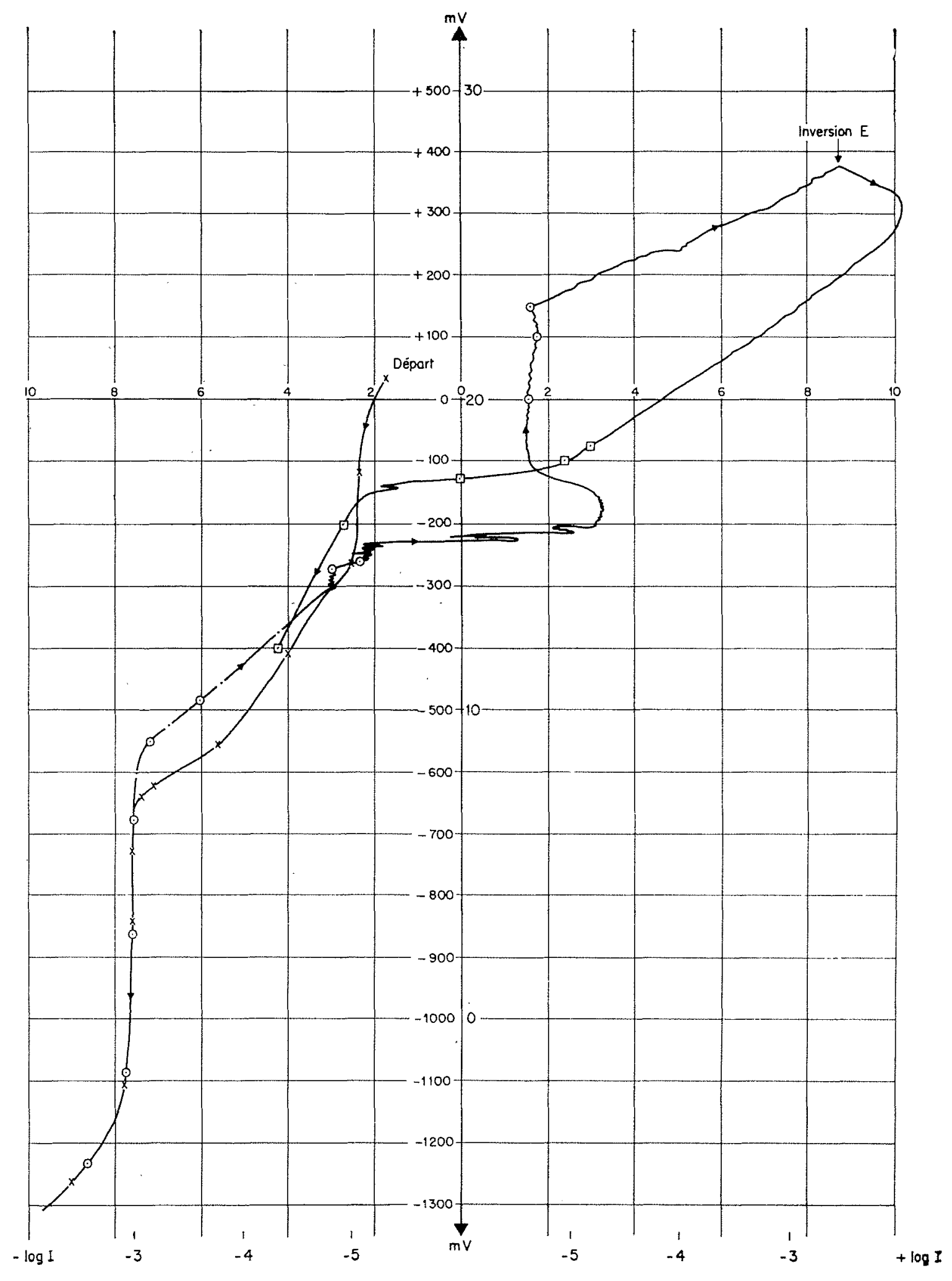

Fig. 10

Courbe potentiocinétique de l'acier austénitique 18-11-3.

Solution aérée. ( $1 \mathrm{~V}$ en $36 \mathrm{mn}$; cellule fermée + circulation; $\mathrm{H}_{2} \mathrm{O}+\mathrm{CINa}: 27,5 \mathrm{~g} \%$; poli : spéculaire.) 


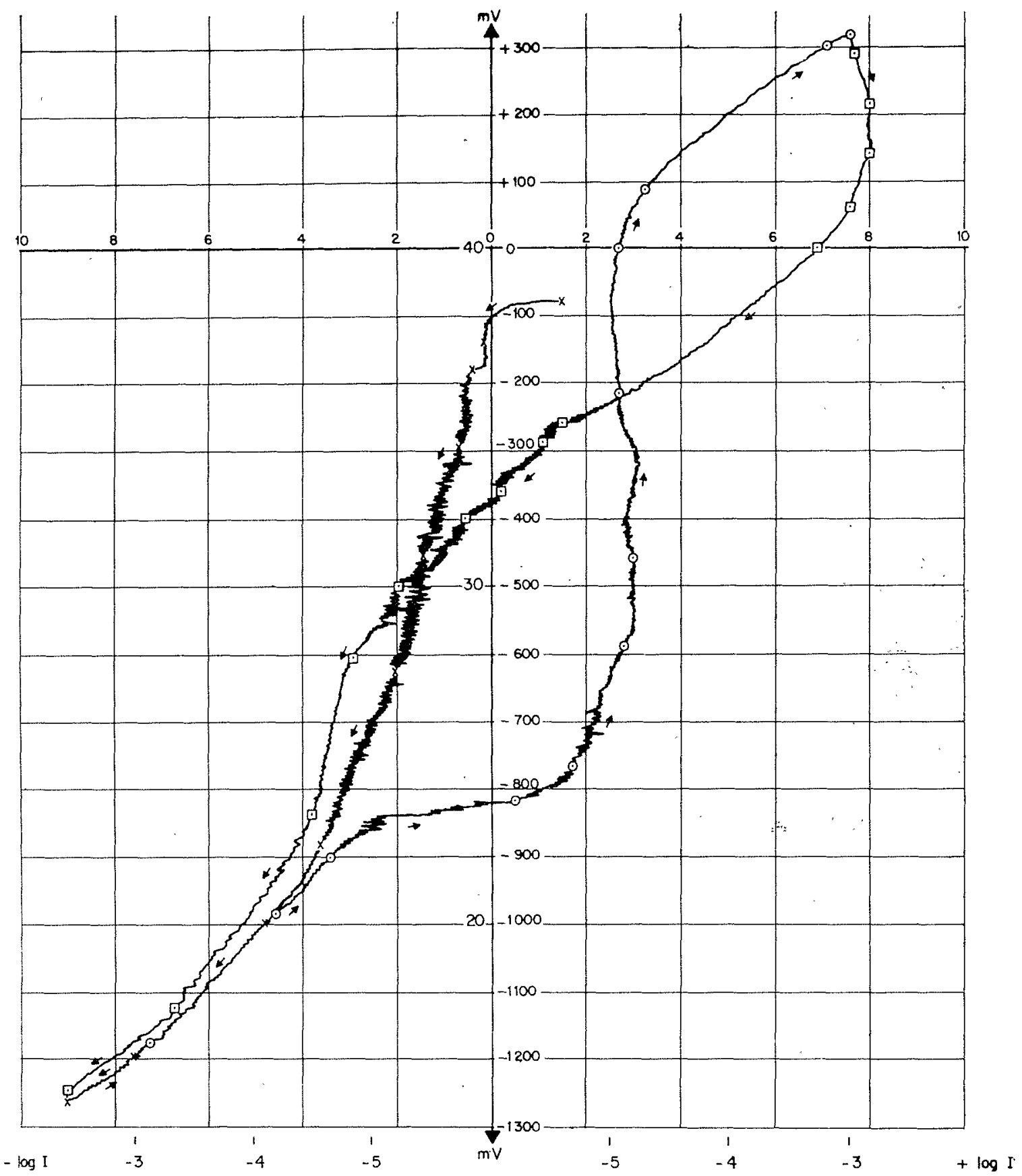

FIG. 11

Courbe potentiocinétique de l'acier austénitique 18-11-3.

Solution désaérée. ( $1 \mathrm{~V}$ en $36 \mathrm{mn}$; cellule fermée + circulation;

$\mathrm{H}_{2} \mathrm{O}+\mathrm{ClNa}: 27,5 \mathrm{~g} \%$; désaéré à l'azote pur; poli : spéculaire.)

d'immunité où la vitesse de corrosion ne saurait être que nulle, ces diagrammes ne fournissent pas d'indications sur la cinétique des réactions.

D'autre part, les diagrammes de Pourbaix sont limités aux réactions des corps simples avec l'eau. Ils n'ont pas encore pu être calculés pour les alliages.
Il fallait donc, pour étudier les cas de corrosion de métaux commerciaux et d'alliages, associer à l'étude des réactions prévisibles par les diagrammes d'équilibre thermodynamique, une méthode fixant l'intensité des réactions pour chaque valeur de potentiel considéré.

Le tracé des courbes de polarisation fournit le moyen d'être renseigné à ce sujet. 
La communication présentée lors des journées d'Aix-en-Provence a montré le parti que l'on pouvait tirer d'un tel travail.

Nous avons modifié le mode opératoire précédemment exposé, pour permettre une analyse plus fine des courbes de polarisation.

L'évolution logarithmique, imposée au courant en fonction du temps, a été remplacée par une variation linéaire du potentiel.

En effet, les paramètres tension et courant fixent respectivement la nature des réactions et leur vitesse; afin de pouvoir se livrer plus commodément à l'analyse des courbes de polarisation, il est préférable de réguler le potentiel de l'électrode étudiée au moyen d'un asservissement électronique classique. Le tracé des courbes par cette méthode porte le nom de tracé potentiocinétique.

Les figures 7,8 et 9 présentent le montage réalisé pour ces essais. L'enregistrement de courant en fonction du temps est obtenu au moyen dun potentiomètre électronique modifié pour donner une réponse logarithmique.

Cette disposition permet de mettre en évidence les réactions où le processus de polarisation correspond à la décharge des ions, celle-ci suivant une progression logarithmique.

Le but recherché en traçant ces courbes était à la fois de déterminer le potentiel limite de protection cathodique des alliages inoxydables et d'évaluer le potentiel à partir duquel apparaît le phénomène de corrosion par piqûres.

La mesure du potentiel de protection cathodique a été obtenue en déplaçant le potentiel du métal jusqu'à - $1300 \mathrm{mV}$, valeur correspondant à l'état actif du fer, du chrome et du nickel, puis en revenant vers les valeurs anodiques.

Les figures 10 et 11 représentent un cycle complet cathodique, puis anodique, de l'acier $18 / 11 / 3$, dans une solution de chlorture de sodium à $27,5 \mathrm{gr} \%$ aérée ou dépourvue d'oxygène, par barbotage continu dans l'électrolyte d'azote purifié.

La figure 10 montre qu'au départ le métal poli est spontanément passivé au contact de la solution chlorurée; à $-650 \mathrm{mV}$ le courant est limité par la diffusion de l'oxygène réduit sur le métal; à partir de $-1100 \mathrm{mV}$ l'intensité mesurée représente la somme du courant de réduction de l'oxygène dissous et de celui correspondant au dégagement de l'hydrogène.

En élevant le potentiel, le phénomène est reversible jusqu'à -650 ; vers -300 apparaissent quelques oscillations du courant traduisant peut-être l'apparition de piqûres.

A intensité nulle, l'acier se trouve à un potentiel d'état passif inférieur de $300 \mathrm{mV}$ au potentiel de départ. La passivité acquise après traitement cathodique est donc inférieure en qualité à la passivité spontanée.

Dans le domaine anodique, la passivation se prolonge jusqu'à $-100 \mathrm{mV}$; entre -100 et $+100 \mathrm{mV}$, la passivité est stable; au-delà de cette valeur, le métal est attaqué avec formation de piqûres.

En ce qui concerne la courbe tracée avec électrolyte désoxygéné (fig. 11), on peut remarquer que la partie cathodique du trácé suit la loi correspondant à l'électrolyse de l'eau.

Il est remarquable que le prolongement de la droite cathodique entre -1200 et $-850 \mathrm{mV}$ coupe l'axe des ordonnées à $-650 \mathrm{mV}$, ce potentiel correspondant à la tension de décomposition des ions $\mathrm{H}^{+}$. Au-dessus de $-840 \mathrm{mV}$, la pente diminue brusquement sous l'influence des réactions d'ionisation du métal.

On peut donc considérer que ce potentiel correspond au potentiel limite de protection de l'acier 18-11-3.

Le tableau II donne le potentiel de protection cathodique de quelques aciers, déterminé dans les mêmes conditions.

Signalons que, par cette méthode, nous n'avons pu mettre en évidence le phénomène de pitting constaté sur le groupe de Saint-Malo avant l'application de la protection cathodique sur les aciers 3 et 4 .

TABLEAU II

\begin{tabular}{|c|c|c|c|c|c|c|c|c|}
\hline & $\mathrm{C}$ & $\mathrm{Cr}$ & $\mathrm{Ni}$ & $\mathrm{Cu}$ & Mo & Al & Mn & \\
\hline Acier $2 . \ldots \ldots$ & 0,1 & 13 & 2 & 0,2 & 0,2 & 0,1 & 0,9 & $-880 \mathrm{mV}$ \\
\hline Acier 4. . & 0,1 & 17 & 4 & 4 & 0,1 & & 0,4 & $-860 \mathrm{mV}$ \\
\hline Acier 5 & 0,05 & 15,5 & 4 & 2,5 & 1,5 & & & $-900 \mathrm{mV}$ \\
\hline Acier $6 . \ldots$ & 0,1 & 23 & 8 & 0,5 & 2 & 0,8 & 0,9 & $-900 \mathrm{mV}$ \\
\hline
\end{tabular}




\section{MESURES SUR LE GROUPE DE SAINT-MALO}

Le groupe de Saint-Malo, ayant été équipé d'anodes selon les dipositions représentées par la figure 12, nous avons procédé à deux séries de mesures au cours desquelles le potentiel des différentes parties du groupe et du conduit a été relevé.

L'expérimentation eut lieu en confiant une électrode au calomel, munie d'un câble étanche, à un homme-grenouille. Les points de mesures étaient préalablement définis et repérés.

\section{Mesures sans protection}

Les potentiels, mesurés après un an d'immersion, sont reportés sur la figure 12 .

Ils donnent donc une image fidèle du comportement des différents éléments par rapport à l'eau de mer.

La différence de potentiel entre la ceinture inoxydable et l'anneau de scellement permet, pour cette section de forme relativement simple,

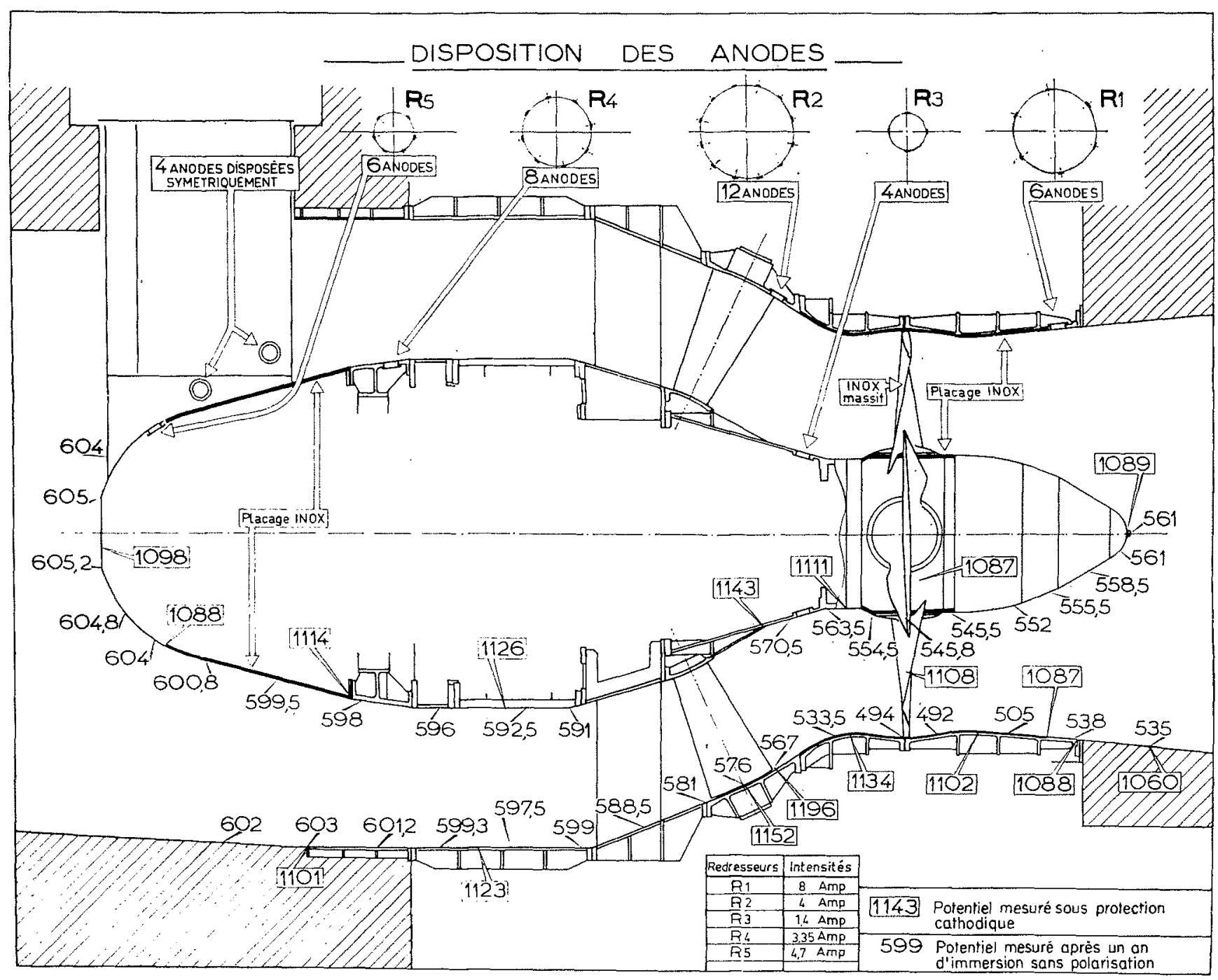

FIG. 12

Mesure du potentiel métal-eau de mer.

599 : potentiel avant protection - 1143 : potentiel sous protection 
de calculer approximativement le courant $\mathrm{du}$ couple électrochimique.

Il atteint 0,63 $\mathrm{A}$. La perte annuelle en acier au carbone de cette section serait done de 5,4 kilos en appliquant les lois de Faraday. Cette attaque ne saurait être exprimée par une diminution d'épaisseur; en effet, la présence de la peinture sur l'acier ordinaire a pour conséquence de reporter l'attaque électrochimique sur les surfaces dépourvues accidentellement de leur revêtement.

\section{Mesures avec protection}

Les mesures de potentiel sous protection cathodique ont été effectuées cinq mois après la mise en service des redresseurs.

Les valeurs de courant et de tension correspondent donc à un état stationnaire de la polarisation.

Le débit total des cinq redresseurs s'élevant à $21,5 \mathrm{~A}$ sous une tension d'environ 8 volts, la puissance dépensée pour la protection d'un groupe atteint $280 \mathrm{~W}$, compte tenu du rendement des redresseurs.

Les potentiels du bulbe sont représentés sur la figure 12 . On peut remarquer que la réparti-

\section{TABleaU III}

Mesures de potentiel sur le conduit amont, effectuées tous les 2 mètres à partir des pales vers la mer jusqu'à la vanne à volets

\begin{tabular}{|c|c|c|c|c|}
\hline $\begin{array}{c}\text { Points } \\
\text { DE MESURes }\end{array}$ & Potentiel & $\Delta^{\prime}$ & $\Delta^{\prime \prime}$ & OBSERYATIONS \\
\hline $\begin{array}{c}1 \\
2 \\
3 \\
4 \\
5 \\
6 \\
7 \\
8 \\
9 \\
10 \\
11 \\
12 \\
13 \\
14 \\
15 \\
16 \\
17 \\
18 \\
19 \\
20 \\
21 \\
\text { Milieu } \\
\text { du volet } \\
\text { inférieur }\end{array}$ & $\begin{array}{ll}1 & 064 \\
1 & 138 \\
1 & 097 \\
1 & 086 \\
1 & 060 \\
1 & 061 \\
1 & 037 \\
1 & 008 \\
& 981 \\
& 958 \\
& 938 \\
& 918 \\
& 903 \\
& 889 \\
& 875 \\
& 864 \\
& 856 \\
& 850 \\
& 844 \\
& 838 \\
& 833 \\
& \\
& 830\end{array}$ & $\begin{array}{r}29 \\
27 \\
23 \\
20 \\
20 \\
15 \\
14 \\
14 \\
11 \\
8 \\
6 \\
6 \\
6 \\
5\end{array}$ & $\begin{array}{l}2 \\
4 \\
3 \\
0 \\
5 \\
1 \\
1 \\
3 \\
3 \\
2 \\
0 \\
0 \\
1\end{array}$ & $\left\{\begin{array}{l}\text { mesures } \\
\text { sur acier }\end{array}\right.$ \\
\hline
\end{tabular}

tion homogène du courant dans le groupe assure un faible gradient de potentiel.

La protection cathodique est donc assuree dans des conditions satisfaisantes.

Les mesures de potentiel ont été étendues aux conduits en béton amont et aval (1); les chiffres relevés ont permis de calculer le courant débité entre les anodes et les structures en béton ainsi que la densité de courant dans ces dernières. Les résultats de cet essai sont reportés tableaux III et IV.

$\Delta^{\prime}$ représente la chute de tension entre deux sections d'électrolyte distantes de 2 mètres;

$\Delta^{\prime \prime}$ correspond à la différence entre deux chutes de tension consécutives $\Delta^{\prime}$.

Si l'on admet que les chutes de potentiel provoquées par la résistance ohmique de l'acier du groupe et des armatures du béton sont négligeables, vu les faibles intensités mises en jeu, il est possible d'estimer le courant de protection collecté par la surface bétonnée.

A une distance de $2,4 \mathrm{~m}$ de l'extrémité du groupe, dans le conduit, le courant est de deux ampères.

On peut assimiler la différence des chutes de potentiel entre deux sections d'électrolyte consécutives $\Delta^{\prime \prime}$ à celle due au courant absorbé par la première de ces sections à partir du groupe.

\section{Tableau IV}

Mesures de potentiels sur le conduit côté bassin, tous les 2 mètres, des pales vers les portes d'écluse

\begin{tabular}{|c|c|c|c|c|}
\hline $\begin{array}{c}\text { Points } \\
\text { De MEsures }\end{array}$ & Potential & $\Delta^{\prime}$ & $\Delta^{\prime \prime}$ & ODSERVATIONS \\
\hline $\begin{array}{r}1 \\
2 \\
3 \\
4 \\
5 \\
6 \\
7 \\
8 \\
9 \\
10 \\
11 \\
12 \\
13 \\
14\end{array}$ & $\begin{array}{r}1067 \\
1062 \\
1009 \\
965 \\
933 \\
910 \\
889 \\
870 \\
854 \\
840 \\
827 \\
818 \\
810 \\
803\end{array}$ & $\begin{array}{r}44 \\
33 \\
23 \\
21 \\
19 \\
16 \\
14 \\
13 \\
9 \\
8 \\
7\end{array}$ & $\begin{array}{r}11 \\
10 \\
2 \\
2 \\
3 \\
2 \\
1 \\
4 \\
1 \\
1\end{array}$ & $\begin{array}{l}\text { mesures } \\
\text { sur acier }\end{array}$ \\
\hline
\end{tabular}

La moyenne de $\Delta^{\prime \prime}$ étant d'environ $2,1 \mathrm{mV}$, le courant correspondant s'élève à $0,15 \mathrm{~A}$, soit $2,1 \mathrm{~mA} / \mathrm{m}^{2}$.

(1) Les faibles gradients de potentiel dans l'eau de mer ne permettant pas une mesure directe de l'intensité, les auteurs ont procédé par la méthode approximative indiquée ci-après. 


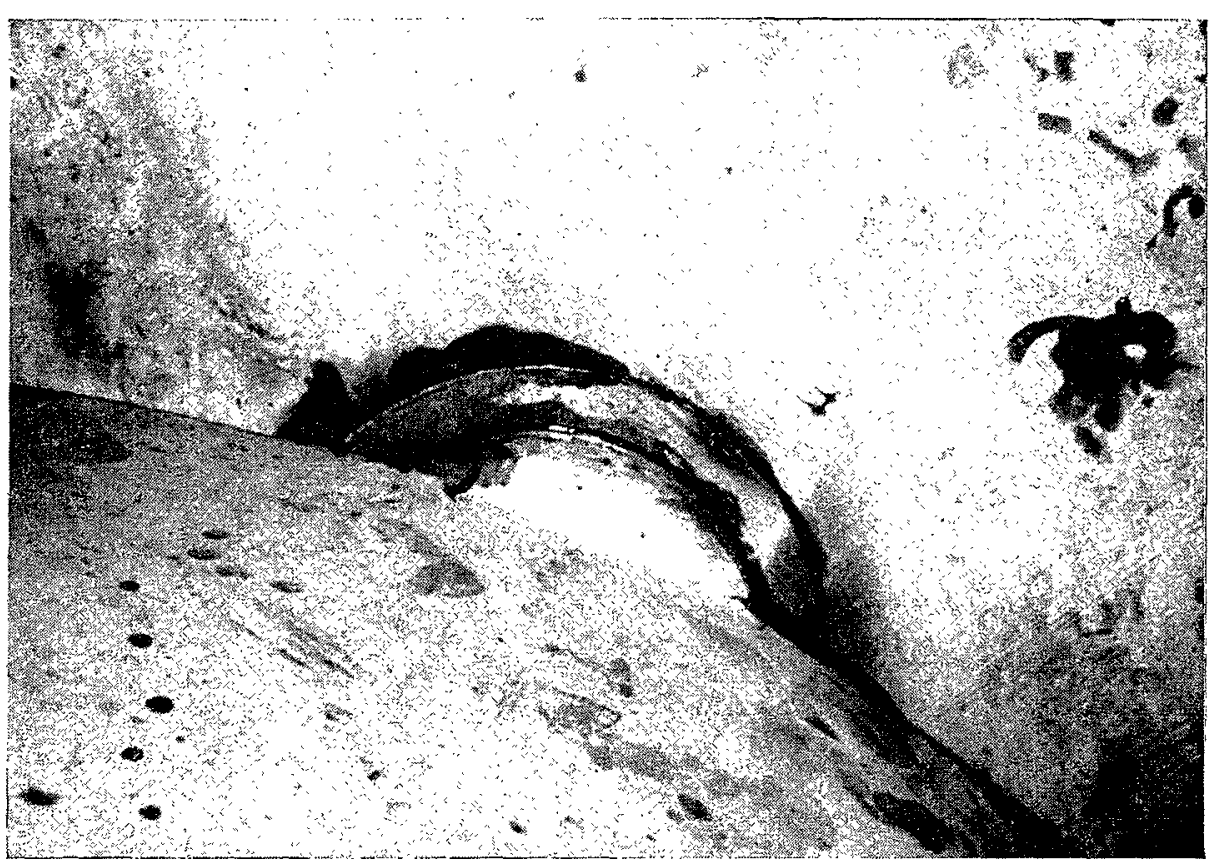

FIG. 13

Tourillon de directrice avant protection cathodique.

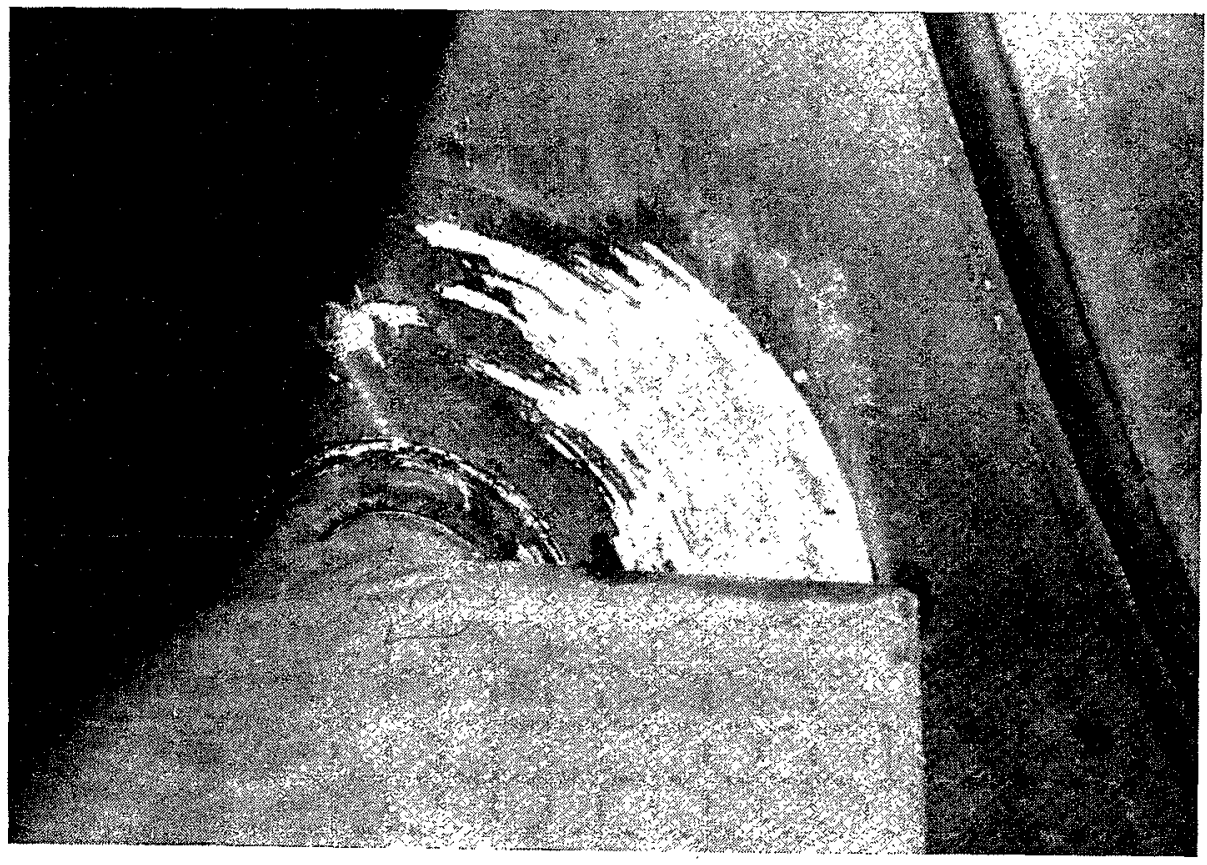

Fig. 14

Tourillon de directrice après application de la protection cathodique. On distingue sur les parties dépourvues de peinture un dépôt protecteur blanchâtre et basique, correspondant à la carbonatation des ions calcium et magnésium. 
Le courant calculé entre les sections correspondant aux points 3 et 4 ressort à $1,4 \mathrm{~A}$.

La densité de courant entre les points 4 et 6 est de $8 \mathrm{~mA} / \mathrm{m}^{2}$ et de $1,6 \mathrm{~mA} / \mathrm{m}^{2}$ au-delà du point 6 .

L'examen du groupe de Saint-Malo montre, après un an de fonctionnement des redresseurs, que la protection cathodique agit efficacement. Sous l'action de la polarisation, les surfaces d'acier doux mises à nu accidentellement se recouvrent d'un dépôt calco-magnésien adhérent et les traces d'oxydes de fer disparaissent.
Les figures 13 et 14 représentent l'état d'un tourillon de directrice avant et après application de la protection. Toutefois, comme l'ont montré les essais effectués entre 1955 et 1957 sur des éprouvettes peintes polarisées, il est recommandé de ne pas abaisser le potentiel des surfaces peintes au-dessous de $-1200 \mathrm{mV}$, la peinture vinylique étant susceptible de cloquer au-delà de ce potentiel. Cette contingence limite à $0,8 \mathrm{~A}$ le courant maximal admissible par anode.

\section{CONCLUSIONS}

Le tracé des courbes potentiocinétiques a permis de chiffrer le seuil de protection cathodique des aciers inoxydables.

Par contre, leur comportement sous l'influence de faibles polarisations, que celles-ci proviennent de couples galvaniques ou de sources extérieures de courant, n'est pas encore parfaitement défini.

Il est donc nécessaire de prendre toutes précautions pour éviter de placer ces métaux dans un état intermédiaire entre la protection cathodique et la passivation.

Les expériences que nous poursuivons permettront, peut-être, d'apporter quelques éclaircissements sur ce sujet.

L'ensemble des essais qui viennent d'être exposés montrent que la protection cathodique d'ouvrages complexes tels que les groupes bulbes, doit tenir compte des impératifs dus à la présence d'acier inoxydable, et à la stabilité des peintures.

Il convient donc de placer les potentiels de toutes les parties de l'ouvrage entre - 900 et - $1200 \mathrm{mV}$; comme l'ont prouvé les mesures effectuées sur le groupe de Saint-Malo, cette condition est remplie.

Les résultats des examens effectués après fonctionnement, durant un an, de la polarisation cathodique, permettent de confirmer l'efficacité de ce mode de protection. Utilisé en association avec les revêtements de peinture, il apporte une solution économique au problème de la protection anticorrosive d'cnsembles multimétalliques.

\section{I S C US S I ON.}

Président : M. Rath

\begin{abstract}
M. le Président remercie MM. Legrand et Lambert pour leur communication et particulièrement M. LAMBERT qui l'a présentée.

Avant d'ouvrir la discussion, M. Le Président précise qu'à l'heure actuelle, bien que cela ne soit pas le cas pour cette communication, il n'y a pas encore unanimité sur la désignation des phénomènes anodiques et cathodiques et l'on ne sait pas toujours, en l'absence de précision dans le texte, s'il s'agit du sens conventionnel du courant ou de celui correspondant à la circulation des électrons.

M. le Président rappelle le but de l'étude, car cette communication fait suite à celle de M. Licheron.

L'acier ordinaire s'attaque au contact de l'eau et l'on peut, en modifiant le potentiel du fer ou de l'acier ordinairc, empêcher cette attaque. La surface des aciers inoxydables est constituée par une couche d'oxydes empêchant les phénomènes électrochimiques correspondant à la corrosion. Il est donc autant logique de
\end{abstract}

faire de la protection cathodique pour des aciers ordinaires, qu'hétérodoxe de la faire sur des aciers inoxydabies.

Dans le cas de la Rance, il est inconcevable de faire les groupes bubes uniquement en acier inoxydable. On doit donc utiliser des aciers ordinaires; ceux-ci, protégés par des peintures, ne sont pas exempts de corrosion, car les peintures sont perméables a quantité de corps agressifs. Il est done logique d'étudier la protection calhodique, mais celle-ci dépassive lacier inoxydable et l'on doit à son tour le protéger, car l'acier inoxydable dépassivé est. plus corrodable que l'acier ordinaire. Les études qui ont été faites par les autcurs de la présente communication et qui ont permis de mener à bien les essais sur éprouvettes, puis sur le groupe G.E.M., puis sur le groupe réel dans l'écluse de Saint-Malo, ont fait progresser la technique sur le plan international.

M. VASSal estime que les aciers inoxydables sont 
sujets au pitting seulement à l'état passif. En zone cathodique, ils risquent une corrosion généralisée faible sans pitting.

M. LAMBERT répond qu'il est d'accord avec M. VASSAL et que leurs avis qui paraissaient contradictoires, concordent en réalité, parce qu'en présence d'oxygène, l'acier inoxydable n'est dépassivé que très bas, comme l'ont montré les courbes potentiocinétiques de l'acier austénitique. M. LAMBErr a essayé de déterminer le potentiel de transition entre l'état actif et l'état passif et il semble que ce potentiel soit très bas comme l'a montré Rocha. Donc, même dans des domaines polarisés cathodiquement de l'ordre de $-500,-600 \mathrm{mV}$, on tronve encore un acier passif, mais pas nécessairement stable, car, en milieu réducteur, on diminue la concentration en $\mathrm{O}_{2}$. Pour qu'il $\mathrm{y}$ ait pitting, il faut qu'il $\mathrm{y}$ ait a la fois du chlore et de l'oxygène.

M. Lambert ne sait pas si l'hypothèse qu'il a émise concernant le maximum à $40 \mathrm{~mA}$ peut être généralisée; il faudrait une expérimentation beaucoup plus importante que celle qui a été effectuée, bien que jusqu'ici le phènomène concorde avec l'hypothèse émise.

M. Vassal demande quelle est la forme de corrosion remarquée sur les éprouvettes qui présentaient un maximum de perte.

M. Lambert répond qu'en particulier Ies éprouyettes a $13 \%$ de chrome étaient atteintes de pitting.

M. le Président demande si les études de protection cathodique qui ont été faites pour le groupe donnent tout apaisement en ce qui concerne le béton ou si l'on peut craindre qu'il $y$ ait des répercussions de la protection cathodique sur la tenue des fers dans le béton.

M. LAMBER's répond qu'il n'a pas travaillé particulièrement ce problème, celui-ci devant être traité par les Services de génie civil à Dinard.

M. le Président pense que si le béton n'est pas d'excellente qualité et si l'électrolyte peut venir plus ou moins librement imprégner les fers, on retrouve un problème de tenue des fers à béton dans l'eau de mer. Or, leur potentiel peut être modifié par la protection cathodique.

M. Berthier donne des explications à ce sujet, car il est, avec le service qu'il dirige, spécialiste de cette question. Il a fait lui-même, en 1941, la première protection cathodique de béton armé qui ait été faite dans le monde, et depuis il a fait rẻaliser de nombreuses protections de grands ouvrages, y compris des ouvrages à la mer.

A première vue, on ne voit pas pourquoi il faut protéger électriquement les armatures de béton armé, puisqu'elles se trouvent dans un milieu de chaux fortement alcalin. Malheureusement, ce milieu alcalin est aussi, par lui-même, salin, et l'alcalinité disparaissant progressivement au cours du temps, le rapport des anions agressifs aux anions inoffensifs croît sans cesse. De plus, par diffusion simple ou par transport électrolytique, les anions agressifs du milieu extérieur pénètrent peu à peu dans le béton, et le fer des armatures finit par se trouver placé dans une sorte de roche calcaire, non alcaline et assez saline, de compacité irrégulière et d'épaisseur faible, et la corrosion, une fois amorcée en un point, tend à progresser et à se généraliser.

La protection cathodique corrige ce processus inéluctable de dégradation en reconstituant d'une part un milieu alcalin passivant autour des armatures, et d'autre part en évacuant les anions salins plus vite que la diffusion ne peut les amener sur le fer. Les principes de cette protection sont simples, leur application est toutefois un art qui exise beaucoup d'expérience. Les installations sont très peu couteuses, si elles ont été prévues dès le projet do l'ouvrage.
M. Berthier tient à prévenir une objection récemment développée. Quelqu'un a dit que la protection cathodique appliquée aux armatures pouvait entrainer la fragilisation par l'hydrogène. Cette annonce a proroqué quelque émotion dans le monde des constructeurs, mais il vaut la peine de donner les apaisements nécessaires sur ce sujet.

On appelle fragilisation par l'hydrogène, une modification de la structure métallique par insertion d'ions hydrogène, c'est-à-dire de protons, dans cette structure, avec modification locale des caractéristiques mécaniques du métal et fragilité apparente des régions ainsi modifiées.

Précisons bien, car la nomenclature est asseż ambiguë, qu'il s'agit ici d'ions normaux, c'est-à-dire d'atomes simplements dépouillés de leurs électrons de valence.

Par contre, les ions gazeux et les ions électrolytiques sont des individus plus complexes, formés d'un ion normal qui se trouve entouré d'une enveloppe compacte de molécules neutres attirées et agglutínées autour de l'ion central par interaction électromagnétique et, en première approximation, électrostatique. L'ion central est réellement séquestré par cette enveloppe qu'on appelle cortège ou auréole d'ionisation, ou, dans le cas de l'électrolyse aqueuse, d'hydratation ou de solvatation. Pour rompre cette enveloppe, il faut dépenser un travail souvent important, par exemple plusieurs électrons-volts pour l'ion hydrogène électrolytique. Ce travail de rupture est pratiquement appelé une surtension.

Revenons à la fragilisation hydrogène. Dans quelles conditions un milieu électrolytique peut-il fournir au métal des protons?

D'abord, il faut que ce milieu contienne des ions électrolytiques hydrogène en concentration suffisante, autrement dit, il faut que ce milieu soit acide, c'està-dire à un pH bas.

Ensuite, il faut pouvoir dépenser le travail de séquestration, c'est-à-dire la surtension électrolytique.

Dans un milieu électrolytique très acide, par exemple dans un bain de décapage acide utilisé en tréfilerie, l'attaque chimique du métal par l'acide consiste en un échange des ions fer normaux du métal et des ions hydrogène séquestrés du liquide. Les ions hydrogène ont alors deux possibilités: ou bien ils s'incorporent au réseau métallique sous forme de protons, ou bien ils s'unissent deux à deux en formant avec deux électrons disponibles du métal une molécule neutre d'hydrogène gazeux, qui se dégage sous forme de bulles.

La première de ces deux transitions ne peut se produire pratiquement qu'en milieu très acide; sa probabilité décroît rapidement dès que le $\mathrm{pH}$ dépasse 2 ou 3 .

Dans la corrosion banale du fer, en milieu peu acide, il doit exister une certaine fragilisation.

On observe alors effectivement une fixation notable d'ions protons dans le milieu métallique avec apparition d'une certaine fragilité. On comprend intuitivement cette fragilité : la structure solide du type métallique comporte un réseau d'ions métalliques identiques et positifs noyés dans un brouillard d'électrons libres. Contrairement aux structures du type ionique et du type covalent, une perturbation dans la distribution des ions métalliques ne change pas beaucoup leur énergie de liaison, d'où la malléabilité. Mais l'injection de protons positifs très petits et capables de fortes interactions, forme, au contraire, des singularités de structure dont les effets ressemblent à une diminution de malléabilité ou une augmentation de la fragilité. Il se produit d'ailleurs en même temps toutes sortes de choses singulières, par exemple une variation des propriétés magnétiques, la conductibilité par porteurs positifs au lieu des porteurs négatifs normaux, etc.

Ce type d'altération se guérit spontanément avec le temps et par un recuit. Un fi] d'acier décapé à froid à l'acide doit être recuit avant de subir un nouveau tréfilage. 
Dans le cas des solutions acides, la fragilisation hydrogène augmente si on place le fer en position cathodique d'électrolyse. Dans ce cas, on augmente la probabilité que les ions hydrogène pénètrent dans le métal au lieu de se dégager sous forme de bulles. Autrement dit, le décapage électrolytique en bain acide, s'il permet d'utiliser des bains moins acides, est plus dangereux que le décapage chimique.

Quand le $\mathrm{pH}$ de la solution augmente, la fragilisation disparaît en même temps que les ions d'hydrogène. Dans une solution de chaux ou de soude, à $\mathrm{pH}$ au-dessus de 9 , on ne peut pas observer avec le fer la moindre fragilisation. Cela est aisé à comprendre, car, par définition, la concentration des ions hydrogène $y$ vaut moins de $10^{-9}$. Dans de tels milieux, neutres ou alcalins, les ions positifs majoritaires sont des ions de métaux alcalins, et ils se substituent complètement aux ions hydrogène pour le transport du courant.

L'enveloppe de séquestration des ions alcalins crève bien plus facilement que celle des ions hydrogène, qui ne prennent plus, dès lors, aucune part an transport de l'électricité.

Corrélativement, le décapage électrolytique des fers, en position cathodique et en solution alcaline, ne donne jamais lieu a aucune fragilisation, sous les plus hautes densités de courant. On peut toutefois observer, à chaud et avec de fortes densités, et en position anodique, une dissolution du fer, mais ce phénomène est tout à fait en dehors de la question.

En définitive, la fragilisation hydrogène ne peut être invoquée contre la méthode d'anticorrosion par polarisation cathodique; elle ne peut apparaitre que dans des conditions extrêmement éloignées de celles où évolue nécessairement cette méthode de protection à laquelle d'ailleurs on ne peut, sur le plan pratique, imputer aucun échec à ee jour.

M. le Président remercie M. Benthier pour son intervention.

M. Duffaut demande, puisque les difficultés de la protection cathodique viennent de l'emploi simultané d'aciers ordinaires et d'aciers spéciaux, si l'on ne pourrait pas songer à revenir uniquement à l'acier ordinaire pour le groupe de la Rance.

M. le Président répond que le choix des matériaux n'est pas dicté par leurs seules propriétés de résistance à la corrosion, mais aussi par leurs caractéristiques mécaniques.

La protection cathodique serait peut-être insuffisante dans les zones de la machine soumises à de grandes vitesses d'eau. Les constructeurs n'ont pas cru bon de retenir la solution de la protection cathodique totale sur un ensemble de machines construit entièrement en matériaux facilement consommables par l'eau de mer.

M. le Président remercie MM. Legrand et Lambert.

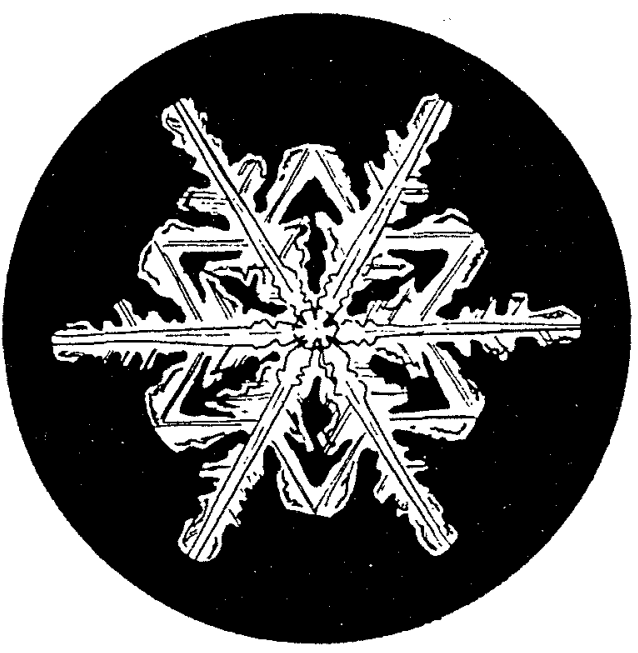

